Proyecciones

Vol. 18, No 2, pp. 137-144, December 1999

Universidad Católica del Norte

Antofagasta - Chile

\title{
A NOTE ON THE NEWTON METHOD FOR DEGREE FOUR POLYNOMIALS *
}

\author{
SERGIO PLAZA S. \\ Universidad de Santiago de Chile, Santiago - Chile
}

\begin{abstract}
We study the Newton method for finding roots of real polynomial equations of degree four from a global and a dynamics point of view. We describe some representative families of four degree polynomials that contain all of the significative features of the dynamics.
\end{abstract}

*Part of this work was supported by Fondecyt Grants \#1941080 and \#1961212, and by Dicyt Grant \#9433 P.S. 


\section{Introduction}

Given that $f$ is differentiable, the Newton method is a first candidate for a numerical solution of the equation $f(x)=0$.

Let $x_{0} \in \mathbf{R}$ be an initial guess for a zero of $f$. Compute

$$
x_{n+1}=N_{f}\left(x_{n}\right)=x_{n}-\frac{f\left(x_{n}\right)}{f^{\prime}\left(x_{n}\right)}, \quad n=0,1,2, \cdots
$$

which defines a discrete dynamical system called the Newton method associated to $f ; N_{f}$ will be called the Newton map (also associated to $f$ ). The orbits of the dynamics of the Newton method are attracted to stable fixed points. Typically, the sequence $\left(x_{n}\right)_{n=0,1,2, \ldots}$ will converge to a zero $x^{*}$ of $f$ and the local convergence is of second order, if $x^{*}$ is a zero of $f$ such that $f^{\prime}\left(x^{*}\right) \neq 0$. The Newton method appears repeatedly in numerical analysis and in discrete dynamical systems literature (see references in [3]). Barna (1953) proved that, for real polynomials of degree $n, n \geq 4$, such that all roots are real and distinct, the set of exceptional points of the Newton method, where Newton map under iteration fails to converge to a fixed point which corresponds to a root of $f$, is homeomorphic to the union of a Cantor set and a countable set. The countable set arises from a Cantor process that is driven by the existence of two or more 2-cycles of the Newton method. Cubic polynomials with three distinct roots have a Newton map with a single 2-cycle; in this case the exceptional set is a countable set. Quadratic polynomials with two distinct roots have a Newton map with trivial dynamics.

We study the dynamical behavior of the Newton method for polynomials of degree four based on the description of generic families of four degree polynomials given in [2].

\section{Basic Features on the Newton Method and Results for Two and Three Degree Polynomials}

We give a brief revision of basic features on the Newton method as well as results on the dynamics of the Newton map for two and three degree polynomials.

Let $f: \mathbf{R} \rightarrow \mathbf{R}$ be a $C^{r}$ function, $r \geq 1$. The Newton map associated to $f$ is

$$
N_{f}(x)=x-\frac{f(x)}{f^{\prime}(x)}, \quad \text { when } f^{\prime}(x) \neq 0
$$


Since $N_{f}^{\prime}(x)=\frac{f(x) f^{\prime \prime}(x)}{\left(f^{\prime}(x)\right)^{2}}$, we have that critical points of $N_{f}^{\prime}(x)=0$ are either roots or inflection points of $f$. We also have that $N_{f}(x)=x$ if and only if $f(x)=0$; that is, the fixed points of $N_{f}$ are the roots of $f$. On the other hand, if $x_{0}$ is a fixed point of $N_{f}$, we have that $N_{f}^{\prime}\left(x_{0}\right)=\frac{m-1}{m}$ where $m$ the multiplicity of the root $x_{0}$ of $f$. Thus if $x_{0}$ is a simple root of $f$, then $x_{0}$ is a superattractive fixed point of $N_{f}$; that is, $N_{f}^{\prime}\left(x_{0}\right)=0$. Therefore the convergence of the iterated $N_{f}^{n}(x)=N_{f} \circ N_{f} \circ \cdots \circ N_{f}(x)$ ( $n$ times) is at least quadratic in a neighborhood of $x_{0}$.

We recall that a topological conjugacy between two maps $f, g: \mathbf{R} \rightarrow \mathbf{R}$ is a homeomorphism $\tau: \mathbf{R} \rightarrow \mathbf{R}$ such that $f \circ \tau=\tau \circ g$. It is well known that conjugacies preserve all significative features of the dynamics.

For two and three degree polynomials we have:

1.- Let $f(x)=a x^{2}+b x+c, a \neq 0$, be a quadratic polynomial. If $f$ has two distinct roots, then $\tau(x)=2 a x+b$ is a topological conjugacy between $N_{f}$ and $N_{q}$, where $q(x)=x^{2}-A$ and $A=b^{2}-4 a c$. Also, we have that $\left.\mathcal{W}^{s}(-\sqrt{A})=\right]-\infty, 0\left[\right.$ and that $\mathcal{W}^{s}(\sqrt{A})=10, \infty[$, where $\mathcal{W}^{s}(p)=\left\{x \in \mathbf{R}: \mathbf{N}_{\mathbf{f}}^{\mathbf{n}}(\mathbf{x}) \rightarrow \mathbf{p}\right.$ as $\left.\mathbf{n} \rightarrow \infty\right\}$ is the attraction basin of the attractive fixed point $p$. And if $f$ does not have real roots, then $N_{q}$ has a chaotic behavior in ] $-\infty, 0[\cup] 0, \infty[$ (see [1]).

2.- Let $f(x)=a x^{3}+b x^{2}+c x+d, a \neq 0$, be a cubic polynomial. Then

$$
N_{f}(x)=\frac{2 a x^{3}+b x^{2}-d}{3 a x^{2}+2 b x+c} .
$$

Let $C=2 b^{3}-9 a(b c-3 a d)$, and $B=9 a c-2 b^{2}$. Let $g(x)=$ $x^{3}+B x+C$, and $\tau(x)=3 a x+b$. Then $\tau \circ N_{f}=N_{g} \circ \tau$; that is, $N_{f}$ and $N_{g}$ are topologically conjugated by the conjugacy $\tau$.

Now let $p(x)=x^{3}+\frac{b}{a} x^{2}+\frac{c}{a} x+\frac{d}{a}$. Then $N_{p}(x)=N_{f}(x)$. Let $f(x)=x^{3}+a x+b^{3}, b \neq 0$, and let $g(x)=x^{3}+c x+1$, where $c=\frac{a}{b^{2}}$. Then the homeomorphism $\tau(x)=\frac{x}{b}$ is a conjugacy between $N_{f}$ and $N_{g}$.

Summarizing, let $f(x)=a x^{3}+b x^{2}+c x+d, a \neq 0, g(x)=x^{3}+$ $C x+\lambda^{3}$, and $h(x)=x^{3}+D x+1$, where $B=9 a c-3 b^{3}, C=$ $2 b^{3}-9 a(b c-3 a d)$. If $B \neq 0$, let $\lambda=\sqrt[3]{B}$, and let $D=\frac{C}{\lambda^{2}}$. Then the homeomorphism $\tau_{1}(x)=3 a x+b$ is a conjugacy between $N_{f}$ and 
$N_{g}$, and the homemorphism $\tau_{2}(x)=\frac{x}{\lambda}$ is a conjugacy between $N_{g}$ and $N_{h}$. Therefore, if $h(x)=x^{3}+\mu x+1$, where

$$
\mu=\frac{C}{(\sqrt[3]{B})^{2}}=\frac{2 b^{3}-9 a(b c-3 a d)}{\left(\sqrt[3]{9 a c-3 b^{2}}\right)^{2}}
$$

is a parameter, then $N_{h}$ is conjugated to $N_{f}$.

Therefore, in order to understand the dynamics of the Newton map of $f$, it is suffices to understand the dynamics of the Newton map of $h(x)=x^{3}+\mu x+1$.

Now let $k_{\gamma}(x)=x^{3}+\gamma x$, and let $N_{\gamma}(x)=\frac{2 x^{3}}{3 x^{2}+\gamma}$ be its Newton map. Then the dynamics of $N_{f}$ is conjugated to the dynamics of $N_{h}$ provided $b \neq 0$, or to the dynamics of $N_{\gamma}$ provided $b=0$.

Let us now consider the following three maps:

(i) $p_{+}(x)=x^{3}+x$;

(ii) $p_{-}(x)=x^{3}-x$;

(iii) $p_{0}(x)=x^{3}$.

If $\gamma=0, k_{0}(x)=p_{0}(x)$. If $\gamma>0$, setting $\theta=\sqrt{\gamma}$, we have that $k_{\gamma}(x)=k_{\theta}(x)=x^{3}+\theta^{2} x$. Finally, if $\gamma<0$, setting $\theta=\sqrt{|\gamma|}$, $k_{\gamma}(x)=k_{\theta}(x)=x^{3}-\theta^{2} x$. Thus, if $\theta \neq 0$, then $N_{\theta}(x)=\frac{2 x^{3}}{3 x^{2} \pm \theta}$. Let $\tau(x)=\frac{x}{\theta}$. Then $\tau^{-1} \circ N_{p_{ \pm}} \circ \tau(x)=\frac{2 x^{3}}{\theta\left(3 x^{2} \pm \theta^{2}\right)}$; that is, if $k_{\theta_{ \pm}}(x)=x^{3} \pm \theta^{2} x$, the homeomorphism ( $C^{\infty}$ diffeomorphism) $\tau(x)=\frac{x}{\theta}$ is a conjugacy between $N_{\theta_{ \pm}}$and $N_{p_{ \pm}}$(see [1]).

\subsection{Main Results}

Let $P(x)=a x^{4}+b x^{3} x+c x^{2} x^{2}+d x+e, a \neq 0$, be a quartic polynomial. Let $\tau: \mathbf{R} \rightarrow \mathbf{R}$ be given by $\tau(x)=x-\frac{b}{4 a}, c_{0}=-\frac{b}{4 a}$. Then

$$
P_{1}(x)=P \circ \tau(x)=P\left(x-\frac{b}{4 a}\right)=\tilde{b}_{0}+\frac{D_{1}}{8 a^{2}} x-\frac{D_{2}}{8 a} x^{2}+a x^{4},
$$

where $\tilde{b}_{0}=P\left(c_{0}\right), D_{1}=b^{3}-4 a c b+8 a^{2} d$ and $D_{2}=3 b^{2}-8 c a$.

We have the following 
Proposition 1. Let $\tau$ be as above. Then $\tau$ is conjugacy between $N_{P}$ and $N_{P_{1}}$.

\section{Proof.}

$$
\begin{aligned}
\tau^{-1} \circ N_{P} \circ \tau(x) & =\tau^{-1}\left(\tau(x)-\frac{P(\tau(x))}{P^{\prime}(\tau(x))}\right) \\
& =\tau(x)-\frac{P(\tau(x))}{P^{\prime}(\tau(x)) \tau^{\prime}(x)}+\frac{b}{4 a} \\
& =x-\frac{P \circ \tau(x)}{(P \circ \tau)^{\prime}(x)} \\
& =N_{P_{1}}(x) .
\end{aligned}
$$

Proposition 2. Let $\sigma: \mathbf{R} \rightarrow \mathbf{R}, \sigma(x)=c_{1} x, c_{1} \neq 0$, and let $P_{2}(x)=$ $P_{1} \circ \sigma(x)=P_{1}\left(c_{1} x\right)=P\left(c_{1} x+c_{0}\right)$. Then $\sigma$ is a conjugacy between $N_{P_{1}}$ and $N_{P_{2}}$ (i.e., $N_{P_{1}}(x)=\sigma \circ N_{P_{2}} \circ \sigma^{-1}(x)$ ).

Proof. It is a straightforward computation.

Remark. If $f(x)=b \varphi(x)$, where $b \neq 0$ is a constant then $N_{f}=N_{\varphi}$, i.e., the identity map is a conjugacy between $N_{f}$ and $N_{\varphi}$.

Theorem 1. Let $P_{3}(x)=b_{1} P_{2}(x)=b_{1} P_{1}\left(c_{1} x\right)=b_{1} P\left(c_{1} x+c_{0}\right)$. Then

$$
P_{3}(x)=b_{1} P\left(c_{1} x+c_{0}\right)=x^{4}-\frac{D_{2}}{8 a^{2}+c_{1}^{4}} x^{2}+\frac{D_{1}}{8 a^{3} c_{1}^{3}} x+b_{1} P\left(c_{0}\right),
$$

and $N_{P_{2}}=N_{P_{3}}$;

i.e., the following diagram commutes 


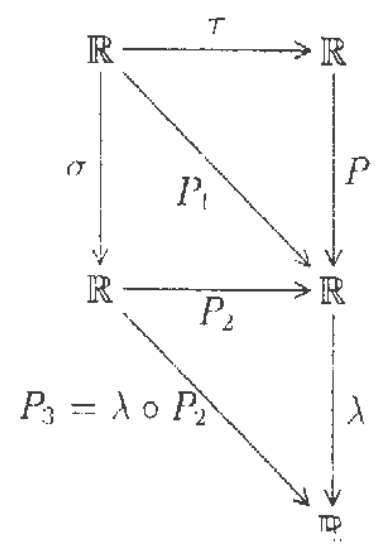

Proof. It is a straightforward computation.

Now for the Newton map associated to each polynomial in the above commutative diagram, we have the following commutative diagram

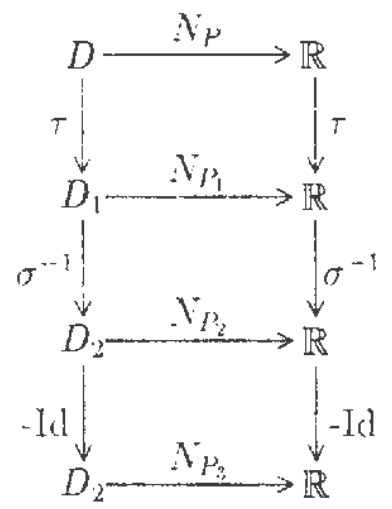


Thus we have proven the following

Theorem 2. Let $P$ and $P_{3}$ be as above. Then $N_{P}$ is conjugated to $N_{P_{3}}$.

We have that any four degree polynomial $P$ may be reduced to

$$
P_{3}(x)=b_{1} P\left(c_{1} x+c_{0}\right)=x^{4}-\frac{D_{2}}{8 a^{2} c_{1}^{2}} x^{2}+\frac{D_{1}}{8 a^{3} c_{1}^{3}} x+b_{0}
$$

Now the dynamics of $N_{P}$ and $N_{P_{3}}$ are conjugated, thus by Theorem 2 it suffices to describe the dynamics of $N_{P_{3}}$. Note that $P_{3}$ depends on two parameters $D_{1}$ and $D_{2}$, and hence we have the following families of polynomials of degree four whose Newton maps contain all of the significative features of the Newton maps of four degree polynomials.

(1) If $D_{1}=D_{2}=0$, then $P_{3}(x)=x^{4}+b_{0}$;

(2) If $D_{1}=0$ and $D_{2}>0$, choosing $c_{1}=\sqrt{\frac{D_{2}}{8 a^{2}}}$, we obtain $P_{3}(x)=$ $x^{4}-x^{2}+b_{0}$;

(3) If $D_{1}=0$ and $D_{2}<0$, choosing $c_{1}=\sqrt{-\frac{D_{2}}{8 a^{2}}}$, we obtain $P_{3}(x)=$ $x^{4}+x^{2}+b_{0}$

(4) If $D_{1} \neq 0$, choosing $c_{1}=\frac{\sqrt[3]{D_{1}}}{2 a}$, we obtain $P_{3}(x)=x^{4}+r x^{2}+x+b_{0}$, where

$$
r=-\frac{D_{2}}{2 \sqrt[3]{D_{1}^{2}}}
$$

is a parameter. 


\section{References}

1] Holmgren, R. A First Course in Discrete Dymamical Systems. SpringerVerlag;: (1994).

2] Movshovitz-Thadar, N., Shmukler, A. Infinitely Many Different, Quartic Polynomiul Curves. The College Mathematics Journal, Vol. 23, $\mathrm{N}^{0} 3$, pp. $186-195,(1992)$.

3] Peitgen. H. (ed.) Neuton, Method and Dynamical Systems. Kluwer AcaAenic Publishers: pp. 54-58, (1989).

Received: Decomber 12, 1998.

\section{Sergio Plaza}

Departamento de Matemáticas y Ciencias de la Cornp.

Universidad de Santiago de Chile.

Casilla 307

Correo 2

Suntiago

Chile 